\title{
Special Issue: Experimental Algorithms
}

The 10th International Symposium on Experimental Algorithms (SEA 2011), previously known as the Workshops on Experimental Algorithms (WEA), took place from May 5 to May 7, 2011 in Kolimpari (Chania, Greece). The WEA/SEA Symposia serve as an international research venue, specifically for design, analysis, and experimental evaluation and engineering of algorithms; the symposia also serve as fora for computational optimization and its applications.

Previous conference locations for SEA include Ischia (2010), Dortmund (2009), Cape Cod (2008), Rome (2007), Monorca (2006), Santorini (2005), Rio de Janeiro (2004), Monte Verita (2003), and Riga (2002).

This special issue contains ten SEA 2011 conference papers; these were selected from 83 contributed articles and 3 invited articles. Among the ten papers in this issue, one paper is based on a plenary talk. The remaining 36 contributed papers and 2 invited articles were published in a special issue of Lecture Notes in Computer Science (LNCS), volume 6630. The acceptance rate for this special issue of Optimization Letters was $12 \%$, with an overall acceptance rate of $54 \%$ for both the special issue of LNCS and Optimization Letters.

We would like to thank the members of the program committee, the authors, and the reviewers. In particular, the thorough review of all submitted papers contributed greatly to the quality both of the published work and of the conference program.

Finally, we would like to thank the steering committee. The committee allowed us to serve as program chairs of SEA 2011 and delegated to us the responsibilities of selecting the program committee, the conference program, and the papers to be published. We are pleased regarding the excellent papers presented at the conference and we look forward to SEA 2012.

May 2011

Steffen Rebennack, Colorado School of Mines 